\title{
IDENTITE DE LITHOCYSTIS PARGUERAE I. JONES 1971, GREGARINE PARASITE DE SIPUNCULIDE, AVEC FILIPODIUM OZAKII HUKUI 1939
}

\author{
par Jean THÉODORIDÈs \\ Laboratoire d'Evolution des Etres organisés \\ Université de Paris V1, 105, boulevard Raspail, F 75006 Paris
}

I. Jones (1971) décrit sous le nom de Lithocystis parguerae une Grégarine intestinale du Sipunculide Siphonosoma cumanense (Keferstein) provenant de Porto-Rico.

Cet auteur qui n'a vu que les trophozoïtes de ce parasite le place dans le genre Lithocystis Giard sur le seul critère de la présence de poils cuticulaires et en fait une espèce nouvelle.

Il est regrettable que 1 . Jones n'ait pas une meilleure connaissance de la littérature concernant les Grégarines de Sipunculides (1) car il est évident que l'espèce trouvée par lui chez S. cumanense est en fait Filipodium ozakii Hukui 1939.

Cette Grégarine a été décrite en détail chez le même hôte au Japon où nous avons pu la retrouver récemment (août 1974) (2) dans la région de Murozomi (Préfecture de Yamaguchi) et les figures données par Jones correspondent tout à fait à celles du Hukui.

Il convient donc de considérer Lithocystis parguerae comme un nomen nudum et de noter que Filipodium ozakii semble parasiter son hôte Siphonosoma cumanense dans toute son aire de répartition.

\section{Références bibliographiques}

HUKUI (T.), 1939. - On the Gregarines from Siphonosoma cumanense (Keferstein). J. Sci. Hiroshima Univ., 7, 1-23.

JONES (I.), 1971. - Observations of an acephaline Gregarine Lithocystis parguerae n. sp. from the Sipunculid Siphonosoma cumanense of Puerto Rico. Carib. Sci. J., 11, 47-50.

Tuzet (O.) et Ormières (R.), 1965. - Sur quelques Grégarines parasites de Phascolion et Aspidosiphon (Sipunculiens). Protistologica, 1, 43-48.

(1) Il ignore en particulier l'article de Tuzet et Ormières (1965) qui décrivent une seconde cspèce de Filipodium ( $F$. aspidosiphoni)

(2) Nous remercions très vivement nos collègues $H$. et $K$. Hoshide pour leur aimable accueil et pour la photocopie de l'article de Hukui. 